\title{
Erratum: Lattice Dynamics Coupled to Charge and Spin Degrees of Freedom in the Molecular Dimer-Mott Insulator $\kappa-(\mathrm{BEDT}-\mathrm{TTF})_{2} \mathrm{Cu}\left[\mathrm{N}(\mathrm{CN})_{2}\right] \mathrm{Cl}$ [Phys. Rev. Lett. 123, 027601 (2019)]
}

Masato Matsuura $\odot$, Takahiko Sasaki, Satoshi Iguchi, Elena Gati, Jens Müller, Oliver Stockert, Andrea Piovano, Martin Böhm, Jitae T. Park, Sananda Biswas, Stephen M. Winter, Roser Valentí, Akiko Nakao, and Michael Lang

(Received 29 October 2020; published 1 December 2020)

DOI: 10.1103/PhysRevLett.125.239901

In our Letter, we used the damped harmonic-oscillator function [1] as a scattering function for phonon modes:

$$
\frac{\Gamma_{q} \hbar \omega}{\left[\hbar^{2}\left(\omega^{2}-\omega_{q}^{2}\right)\right]^{2}+\left(\Gamma_{q} \hbar \omega\right)^{2}}
$$

However, in the fits to the data, accidentally a modified scattering function which differs from Eq. (1) by its denominator $\left[\hbar\left(\omega-\omega_{q}\right)\right]^{2}+\left(\Gamma_{q} \hbar \omega\right)^{2}$ was used. We corrected the scattering function and show the refitted results in Fig. 1 . The measured data are well reproduced by these new fits.

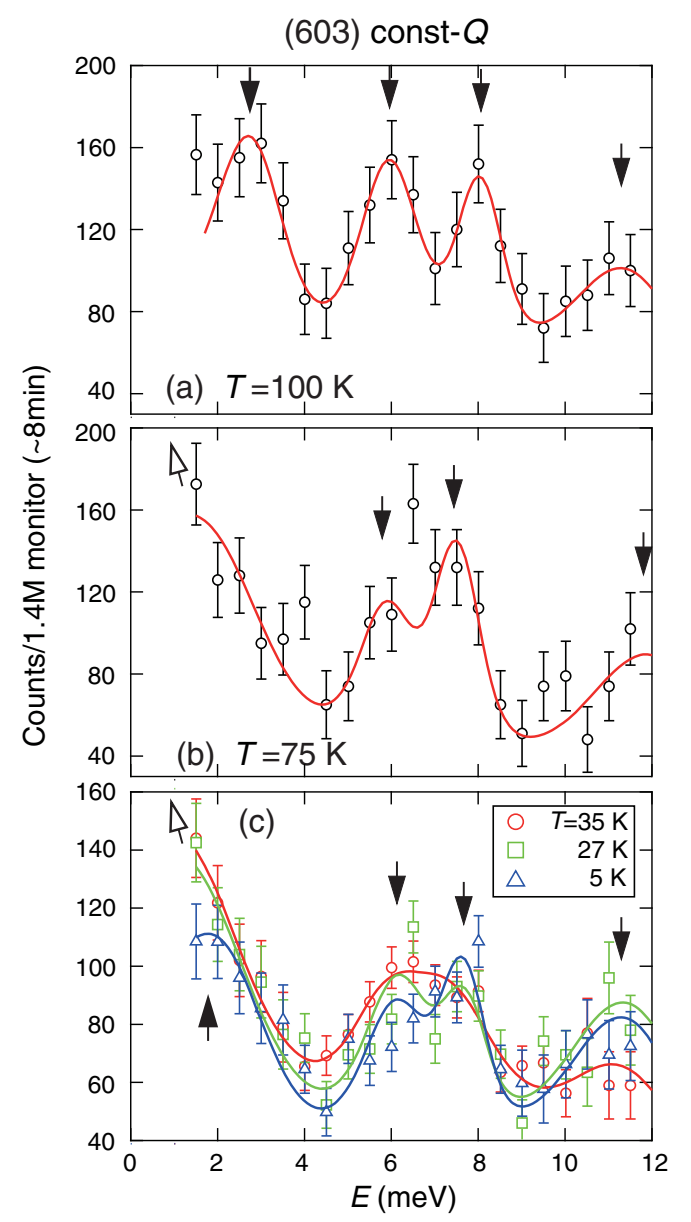

FIG. 1. Corrected Fig. 2 of the Letter. Temperature dependences of constant- $Q$ scans at (603). The solid lines are fits to four damped harmonic oscillator functions at $E \sim 2.8,6,8$, and $11 \mathrm{meV}$. 


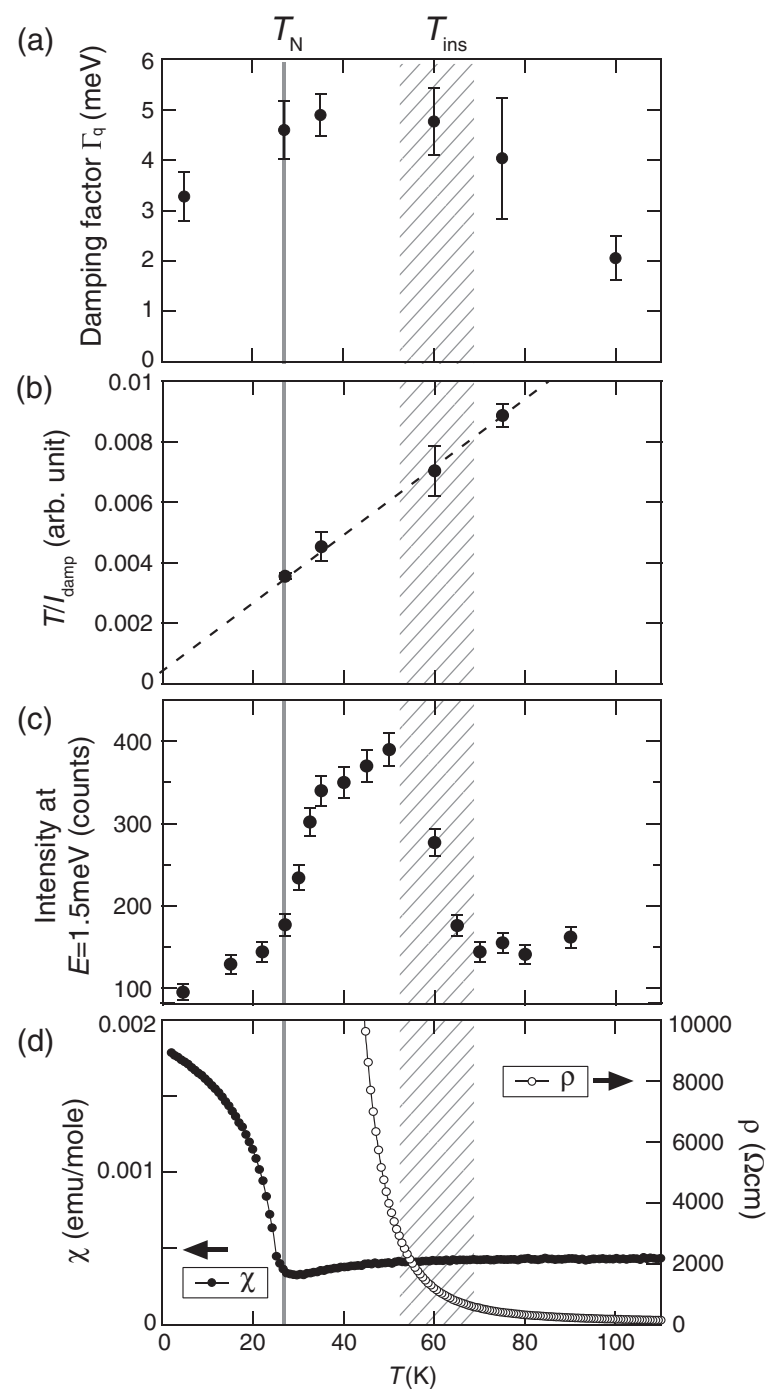

FIG. 2. Corrected Fig. 3 of the Letter. Temperature dependence of (a) the damping factor $\Gamma_{q}$ for the low-lying optical modes at (603), (b) $T / I_{\text {damp }}$ for the low-lying optical modes (see text for details), (c) the intensity at $E=1.5 \mathrm{meV}$ at (603), (d) the out-of-plane electrical resistivity $(\| b)$, and out-of-plane dc magnetic susceptibility $(\| b)$ at $\mu_{0} H=0.5 \mathrm{~T}$.

Figure 2(a), which should replace Fig. 3(a) in the Letter, shows the temperature dependence of the damping factor $\Gamma_{q}$, derived from these fits, for the low-lying optical modes. Although the overall magnitude of the damping factor becomes larger for the corrected fits, the temperature dependences of the damping factors are similar: the damping factor becomes much larger than the phonon energy $(2.8 \mathrm{meV})$ for $27 \leq T \leq 75 \mathrm{~K}$, indicating the strong phonon damping in this temperature range. The conclusions in the Letter remain unchanged by these corrections.

[1] K. Gesi, J. D. Axe, G. Shirane, and A. Linz, Phys. Rev. B 5, 1933 (1972). 